\title{
Measurement of the acoustic-to-optical phonon coupling in multicomponent systems
}

\author{
Antonio Caretta, ${ }^{1, *}$ Michiel C. Donker, ${ }^{1}$ Diederik W. Perdok, ${ }^{1}$ Davood Abbaszadeh, ${ }^{1}$ Alexey O. Polyakov, ${ }^{1}$ \\ Remco W. A. Havenith, ${ }^{1,2}$ Thomas T. M. Palstra, ${ }^{1}$ and Paul H. M. van Loosdrecht ${ }^{1}$ \\ ${ }^{1}$ Zernike Institute for Advanced Materials, University of Groningen, Nijenborgh 4, 9747 AG Groningen, The Netherlands \\ ${ }^{2}$ Ghent Quantum Chemistry Group, Department of Inorganic and Physical Chemistry, Ghent University, \\ Krijgslaan 281 (S3), B-9000 Gent, Belgium \\ (Received 17 April 2014; revised manuscript received 22 December 2014; published 25 February 2015)
}

\begin{abstract}
In this paper we investigate the acoustic-to-optical up-conversion phonon processes in a multicomponent system. These processes take place during heat transport and limit the efficiency of heat flow. By combining time-resolved optical and heat capacity experiments we quantify the thermal coupling constant to be $g \sim$ $0.410^{17} \mathrm{~W} / \mathrm{Km}^{3}$. The method is based on selective excitation of a part of a multicomponent system, and the measurement of the thermalization dynamics by probing the linear birefringence of the sample with femtosecond resolution. In particular, we study a layered multiferroic organic-inorganic hybrid, in the vicinity of the ferroelectric phase transition. A diverging term of the heat capacity is associated to soft-mode dynamics, in agreement with previous spectroscopy measurements.
\end{abstract}

DOI: 10.1103/PhysRevB.91.054111

PACS number(s): $65.60 .+\mathrm{a}$

\section{INTRODUCTION}

Heat transport phenomena, one the oldest topics in thermodynamics, continue to trigger novel research. In the 18th century the problem of heat transport lead to the formulation of the first and second thermodynamical laws (Clausius and Kelvin) and to the early description of the statistical aspects of thermodynamics (Boltzmann-Maxwell) [1,2]. Today, the interesting aspects of the electron and heat transport in graphene [3], ballistic phenomena [4] and so on, still represents one of the most intriguing fields of research. Additionally, the advances of time-resolved experimental techniques allow the investigation of such phenomena with resolution down to the attosecond regime [5]. A large variety of phenomena was discovered involving the nonequilibrium transfer of energy [6], charges, or spins [7]. The description of these processes requires the understanding of the microscopic origin of the propagation of energy. Here we focus on heat transport in insulating paramagnets, thus the role of electrons and phonons is fundamental.

Heat transport in insulators is in general carried by lowenergy acoustic phonons. It is expected that quasiparticles with high group velocity can space-transfer energy very efficiently. The transport due to phonons is carried mostly by $k=0$ acoustic phonons, i.e., at the momentum position where the dispersion curve is steepest. Spontaneous decay to other phonons with higher phase velocity is protected by selection rules [8]. Hot low $k$-vector acoustic phonons travel through the lattice, redistributing energy towards colder regions, until equilibrium is reached. To reach thermal equilibrium the acoustic phonons are up-converted to higher-energy optical phonons, via phonon-phonon scattering mechanisms [9]. Acoustic $(a)$ to optical ( $o$ ) phonon scattering processes, like $a+a \rightarrow o$, cause also a reduction of the transport efficiency of the acoustic phonons since the group velocity of optical phonons is in general lower. It was actually shown that in one dimension the presence of optical phonons can even enhance heat tranposrt

\footnotetext{
*a.caretta@rug.nl
}

[10]. Such up-conversion processes are also extremely relevant for photovoltaic applications, where the cell efficiency can to be enhanced by thermal up-conversion of low-energy phonons [11]. Although the role of phonon scattering is fundamental to understanding thermal equilibration [12], few experimental studies address this issue, possibly because few experiments can specifically investigate such phenomena.

In this paper we investigate the up-conversion phononphonon $a+a \rightarrow o$ scattering mechanisms in a multicomponent system by the use of a variety of experimental methods. In particular, we investigate the layered multiferroic organicinorganic hybrid $\left(\mathrm{C}_{6} \mathrm{H}_{5} \mathrm{CH}_{2} \mathrm{CH}_{2} \mathrm{NH}_{3}\right)_{2} \mathrm{CuCl}_{4}$ in proximity of the ferroelectric phase transition $[13,14]$. We combine timeresolved birefringence (TRB) optical techniques, differential scanning calorimetry (DSC), and denstiy functional theory (DFT) calculations. We quantify the thermal coupling constant describing the interaction between high group velocity (HGV) low-energy phonons and higher-energy optical phonons. We show that the time scales and relaxation times obtained by time-resolved experiments are strongly influenced by changes of the heat capacity, changes that are often observed in proximity of phase transitions. A method, based on the analysis of the partial contribution to the heat capacity, is described to extract fundamental constants from the measured time scales.

\section{EXPERIMENTAL DETAILS}

The layered organic-inorganic hybrid $\left(\mathrm{C}_{6} \mathrm{H}_{5} \mathrm{CH}_{2} \mathrm{CH}_{2}\right.$ $\left.\mathrm{NH}_{3}\right)_{2} \mathrm{CuCl}_{4}$ is a transparent multiferroic insulator. Details of the structure and of the multiferroic properties can be found in Refs. $[13,15]$. Small platelets are formed by slow evaporation from the aqueous solution of 2-phenylethylammonium chloride and $\mathrm{CuCl}_{2} \cdot \mathrm{H}_{2} \mathrm{O}$ salts. The structure consists of corner-sharing $\mathrm{CuCl}_{6}$ octahedra forming chess-board-like sheets (along the $a b$ plane), the empty spaces filled from both sides by organic cations, oriented perpendicularly to the layers $[13,16]$. The crystals are yellow at room temperature and have a large flat surface corresponding to the $a b$ plane perpendicular to the $c$ axis. The ferromagnetic phase transition 


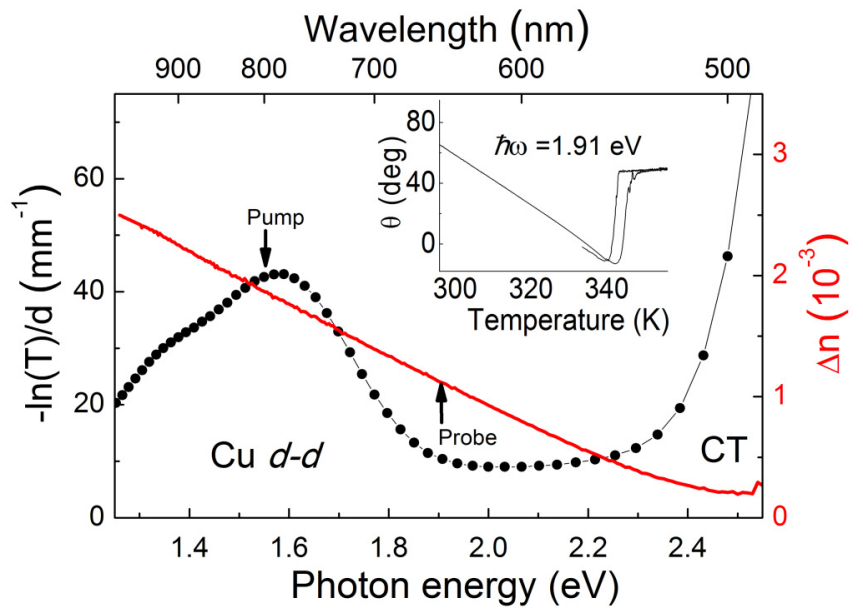

FIG. 1. (Color online) Absorbance $-\ln (T) / d(T$ transmission and $d$ thickness) and birefringence $\Delta n$ of PEACuCl sample at $300 \mathrm{~K}$. The two NIR bands around $1.5 \mathrm{eV}$ are phonon-assisted $\mathrm{Cu} d-d$ transitions and the strong absorption edge at $2.5 \mathrm{eV}$ is the onset of the $\mathrm{Cl}(3 p)-\mathrm{Cu}(d)$ charge transfer $(\mathrm{CT})$ transitions. Inset: Birefringence, measured at $1.91 \mathrm{eV}$, as function of temperature near the ferroelectric phase transition at $340 \mathrm{~K}$.

at $13 \mathrm{~K}$ and the improper ferroelectric phase transition at $T_{C}=340 \mathrm{~K}$ have extensively been described in Refs. [17-19].

Absorbance measurements are performed with the light propagating along the $c$ axis, perpendicularly to the layers, by a standard lock-in technique with a Halogen lamp as source, a mechanical chopper, and a monochromator. Figure 1 shows the recorded absorbance spectra at $300 \mathrm{~K}$. The $\mathrm{Cu} d$ - $d$ transitions are observed as a double-peaked band around 1.2-1.8 eV, whereas the onset of the $\mathrm{Cl}(3 p)-\mathrm{Cu}(3 d)$ charge transfer band is observed at approximately $2.5 \mathrm{eV}$ [14].

Frequency-resolved birefringence measurements are performed using the polarization modulation by a photoelastic modulator and lock-in techniques for detection. The resulting birefringence spectrum at room temperature is shown in Fig. 1 (red line). Temperature-dependent experiments on the birefringence is performed at $1.91 \mathrm{eV}(650 \mathrm{~nm})$, between 280 and $360 \mathrm{~K}$ (see inset in Fig. 1).

As for the absorbance measurements, the samples are placed at a normal angle of incidence. The polarization of the light is chosen at approximately at $45^{\circ}$ from the $a$ axis to maximize the observed birefringence.

Differential scanning calorimetry (DSC) was performed using a DSC Q1000 (TA Instruments) in modulated mode on $\sim 10 \mathrm{mg}$ samples. The heating rate was $1 \mathrm{~K} / \mathrm{min}$, the amplitude of temperature modulation was $0.5 \mathrm{~K}$, and the period of modulation $100 \mathrm{~s}$. The values of $c_{P}$ are calculated via the reverse $c_{P}$ method [20], after calibration with sapphire samples. The specific (mass) heat capacities $c_{P}$ of powder samples and single crystals are in good agreement.

Time-resolved birefringence (TRB) experiments are performed with a 1-kHz repetition rate amplified Ti:Sapphire laser (Hurrican, Spectra Physics). The fundamental of the laser at $\hbar \omega=1.55 \mathrm{eV}(800 \mathrm{~nm})$ is used as a pump. The 120 -fs-long pulses are focused on the sample to a spot with a diameter of $\sim 120 \mu \mathrm{m}$. The probe pulses at $1.91 \mathrm{eV}(650 \mathrm{~nm})$ (a)

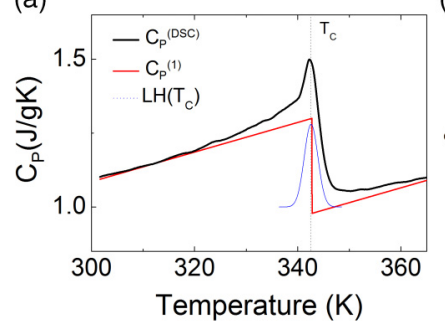

(b)

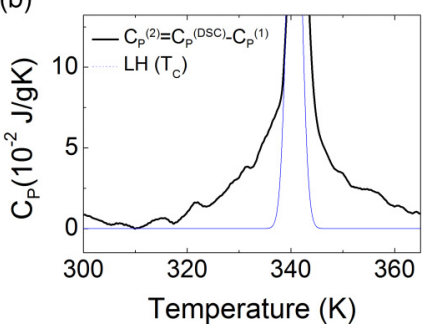

FIG. 2. (Color online) (a) Specific (mass) heat capacity $c_{P}$ near the improper ferroelectric phase transition at $340 \mathrm{~K}$. Three components contribute to $c_{P}$ : a first-order-like step function $c_{P}^{(1)}$, a narrow peak at $T_{C}$ corresponding to latent heat ( $\left.\mathrm{LH}\right)$, and (b) a diverging term $c_{P}^{(2)}$, symmetrically decreasing moving away from $T_{C}$.

are generated using a TOPAS Optical Parametric Amplifier (second harmonic of the signal). A probe polarization ratio $E_{s} / E_{p} \sim 10^{5}$ is obtained using a Glan-Taylor prism, which stretches the pulse to about $\sim 200$ fs. The measurements are performed in transmission, with the sample mounted in a KONTI IT (cryovac) cryostat with temperature stabilization better than $0.2 \mathrm{~K}$. The detection of the probe polarization change is performed using a standard balanced photodetection technique, with synchronous chopping at $500 \mathrm{~Hz}$ (see Ref. [21]).

\section{PRELIMINARY CONSIDERATIONS}

Within 1 ps the pump excitation at $\hbar \omega=1.55 \mathrm{eV}$ predominantly excites the Jahn-Teller $\mathrm{Cu}-\mathrm{Cl}$ inorganic phonon modes. A photon at $1.55 \mathrm{eV}$ hits the center of the phonon-assisted $\mathrm{Cu}$ $d$ - $d$ transitions (Fig. 1). The absorption of a photon implies the creation of phonon modes strongly coupled to the electronic transition, like the $\mathrm{Cu}-\mathrm{Cl}$ Jahn-Teller modes. In analogy to above-band gap optical transitions in semiconductors, each absorbed photon releases an energy $\Delta q \sim 0.25 \mathrm{eV}$, corresponding to the excess photon energy with respect to the band gap $\left(E_{\mathrm{Gap}} \sim 1.3 \mathrm{eV}\right)$. This excess phonon energy is redistributed to the other degrees of freedom, until thermal equilibrium is reached. It should be noted that no, or very few, free carriers are generated in the excitation process. In fact, the analogy with semiconductors serves only as a estimate of the excess energy, while the actual photon absorption mechanism closely resembles Frank-Condon excitation. This microscopic mechanism is supported by the strong insulating character of the hybrid materials in the considered temperature range [22]. For the time scale of the experiment $(<1 \mathrm{~ns})$ spatial heat diffusion and auger recombination can be neglected. The temperature rise $\Delta T_{\infty}$ of the whole system can be written as

$$
\Delta T_{\infty}=n_{\mathrm{abs}} \frac{\Delta q}{C_{P}},
$$

where $C_{P}$ is the heat capacity of the excited volume and $n_{\text {abs }}$ is the number of absorbed photons. The excited volume $V$ is a cylinder having a diameter of $120 \mu \mathrm{m}$ (spot size) and a length corresponding to the penetration depth $\delta_{\omega}=2 / \alpha_{\omega} \sim 40 \mu \mathrm{m}$, where $\alpha_{\omega}$ is the absorbance at $1.55 \mathrm{eV}$ (Fig. 1). Given the room temperature (mass) specific heat capacity $c_{P}=1 \mathrm{~J} /(\mathrm{gK})$ (Fig. 2) and the density $\rho=1.49 \times 10^{3} \mathrm{~kg} / \mathrm{m}^{3}$ we find 


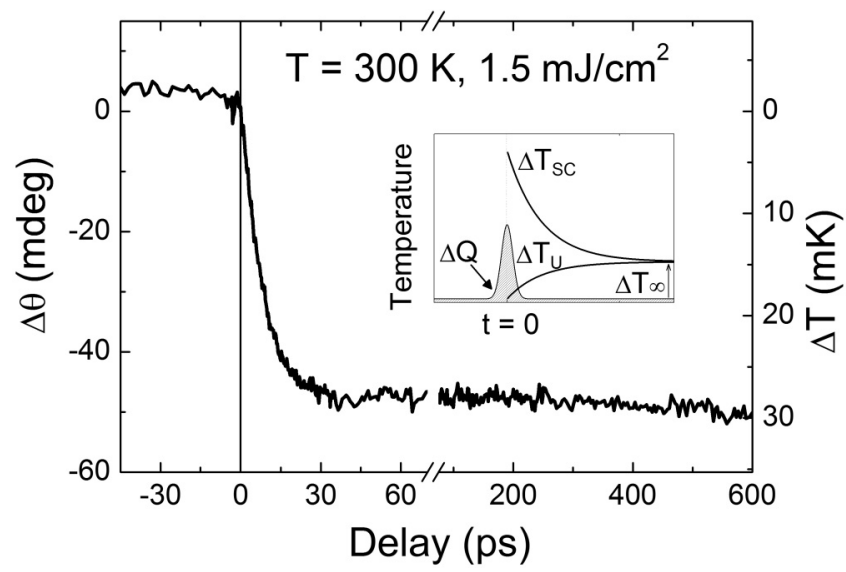

FIG. 3. Time-resolved birefringence $\Delta \theta(t)$ at $300 \mathrm{~K}$ (energy density $1.5 \mathrm{mJcm}^{-2}$ ). The inset figure shows the expected variation of the sub-systems temperatures: strongly coupled (SC) phonons are instantaneously excited by the laser pulse while the unperturbed $(U)$ phonons, initially cold, slowly thermalize with the SC phonons.

$C_{P}=c_{P} \rho V \sim 2.110^{-6} \mathrm{~J} / \mathrm{K}$. Considering a pump pulse energy $\Phi=250 \mathrm{~nJ}$ (which corresponds to $1.5 \mathrm{~mJ} / \mathrm{cm}^{2}$ energy density, $0.1 \%$ of the $\mathrm{Cu}$ ions are excited), and that all photons $n_{\text {Abs }}=\Phi / \hbar \omega$ are absorbed in the volume $V$ excited by the laser, with Eq. (1) it is found that

$$
\Delta T_{\infty} \simeq \frac{\Delta q}{C_{P} \hbar \omega} \Phi \sim 20 \mathrm{mK} .
$$

To instantaneously measure such temperature rise after pump excitation we measured the change in birefringence with femtosecond resolution. In Fig. 3 the change of birefringence angle $\Delta \theta$ is measured with respect to the pump pulse arrival. After the pump arrival $\theta$ starts to decrease exponentially and reaches a minimum $\Delta \theta_{\min } \sim-40 \mathrm{mdeg}$, at approximately 30 ps. As can be observed from the inset of Fig. 1, the birefringence angle $\theta$ is linear with the temperature. Thus it is possible to calibrate the variation of $\theta$ to a temperature variation. In fact, it is possible to write $\theta\left(T+\Delta T_{\infty}\right)=\theta(T)+$ $\frac{\partial \theta(T)}{\partial T} \Delta T_{\infty} \equiv \theta(T)+\Delta \theta_{\infty}$. For temperatures below $T_{C}$ we have

$$
\Delta \theta_{\infty}=\left.\frac{\partial \theta(T)}{\partial T}\right|_{T<T_{C}} \Delta T_{\infty} \sim-\left(1.7^{\circ} / K\right) \Delta T_{\infty} .
$$

According to the estimate of Eq. (2), it is found that $\Delta \theta_{\infty} \sim$ $-34 \mathrm{mdeg}$, in agreement with TRB measurements at late times $\Delta \theta_{\min }$. This indicates that indeed the birefringence $\theta$ probes the temperature of the system, and in particular the temperature of a system component- the "unperturbed" $(U)$ ensemble-which is not instantaneously heated by the pump pulse. In fact, assuming strong coupling (SC) of the phonon modes to the excitation (as shown in the inset of Fig. 3), the temperature $\Delta T_{S C}$ should rise immediately and then decrease. According to this observation it will be more meaningful, from now on, to convert all the TRB transients to $\Delta T_{U}$ thermalization transients, by the use of Eq. (3). The model presented here is a very general model, similar to thermal relaxation of electrons in metals [23,24]. It is fundamental to discuss in this case the degrees of freedom involved in the studied dynamical processes, and this is the goal of the next section.

\section{RESULTS AND DISCUSSION}

Figure 4 shows the transient response [expressed as $\Delta T_{U}(t)$ ], for a variety of temperatures, in the vicinity of the ferroelectric phase transition. The measurements are performed at constant pump fluence $\Phi \sim 250 \mathrm{~nJ} /$ pulse. One clearly observes that, upon approaching $T_{C}$ from below, the thermalization process slows down, and the saturation temperature $\Delta T_{\infty}$ decreases.

The transient response can be understood in terms of a simple two-temperature model. As before, we consider the system as composed by the $\mathrm{SC}$ and $U$ phonons. Both systems are, respectively, described by the temperatures $T_{S C}$ and $T_{U}$ and the heat capacities $C_{S C}$ and $C_{U}$. The two systems are thermally coupled through a phonon-phonon coupling constant $g$. As an initial condition, we assume the instantaneous excitation of the SC component: $\Delta T_{S C}(t=0)=\Delta Q / C_{S C}$. The heat equation describing the heat flow from SC to $U$ is given by

$$
\frac{d Q_{S C}}{d t}=-g\left(T_{S C}-T_{U}\right) .
$$

Since the total system is isolated, at least for the considered time scales, we have $d Q_{S C}+d Q_{U}=0$. By solving Eq. (4) we obtain

$$
\Delta T_{U}(t)=\Delta T_{\infty}\left(1-e^{-\tau t}\right),
$$

where

$$
\Delta T_{\infty}=\frac{\Delta Q}{C_{S C}+C_{U}},
$$

and

$$
\tau=\frac{C_{S C} C_{U}}{g\left(C_{S C}+C_{U}\right)} .
$$

Equation (5) fits very well to the transient data, yielding the temperature dependence of both the thermalization time $\tau$ and equilibration temperature $\Delta T_{\infty}$ as shown in Fig. 4(b). The most remarkable observation is perhaps the divergence of $\tau$, growing, from the 5-ps room temperature value, by (a)

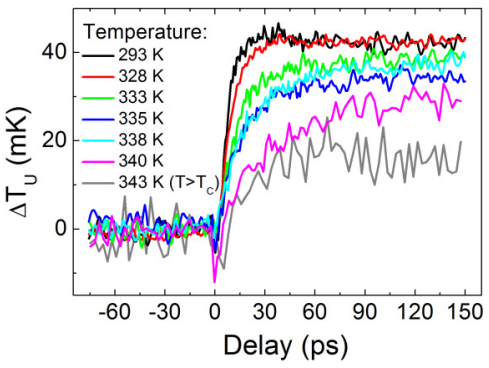

(b)

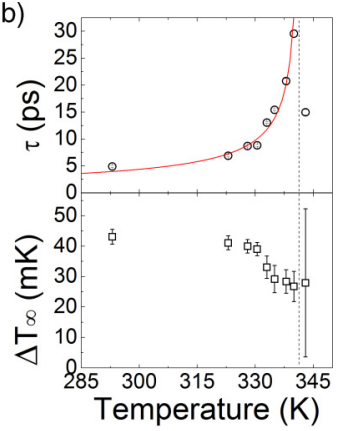

FIG. 4. (Color online) (a) Thermalization dynamics of the unperturbed system temperature $\Delta T_{U}$ in the vicinity of the phase transition at $340 \mathrm{~K}$. (b) Single exponent fit results showing that, as the temperature increases towards $T_{C}$, the relaxation time $\tau$ diverges and the saturation temperature $\Delta T_{\infty}$ slightly decreases. 


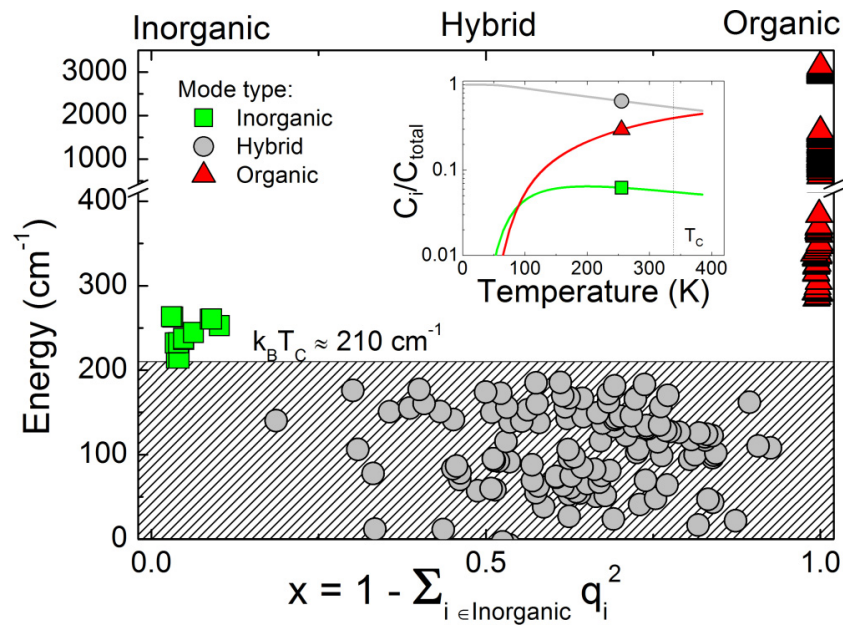

FIG. 5. (Color online) Phonon diagram at the $\Gamma$ point $(\gamma=0)$. Note that $\sum_{i \in \text { Inorganic }} q_{i}^{2}$ represents the normalized mean square displacement of the inorganic ions $\mathrm{Cu}$ and $\mathrm{Cl}$. The inset figure shows the relative contribution to the total heat capacities of the three components as a function of temperature, assuming Einstein phonon heat capacity.

a factor of 5 close to $T_{C}$. The variation of $\Delta T_{\infty}$ is less dramatic, showing a small continuous decrease with increasing temperature. The large error bar of $\Delta T_{\infty}$ for $T>T_{C}$ is caused by the propagation of the large uncertainty of $\left.\frac{\partial \theta}{\partial T}\right|_{T>T_{C}}$. From Eq. (6), since $C_{P}=\left(C_{S C}+C_{U}\right) \equiv C_{\text {total }}$ and $\Delta Q$ is constant (in fact the absorbance at $\hbar \omega=1.55 \mathrm{eV}$ is nearly constant with temperature across the phase transition), we can calculate $C_{\text {total }}$, which is in good agreement with the measured $C_{P}=c_{P} \rho V$ (see Ref. [21]).

We use now the DFT calculations of the phonon frequencies to obtain a precise information of the character of SC and $U$ modes. In Fig. 5 the energy of the vibrational modes at the $\Gamma$ point, calculated as in Ref. [15], along the $y$ axis is shown as a function of $x=1-\sum_{i \in \text { Inorganic }} q_{i}^{2}$, where $q_{i}$ are the normalized mean square displacement-so that $\sum_{i} q_{i}^{2}=$ 1 - of the inorganic ions $(\mathrm{Cu}$ and $\mathrm{Cl})$. The resulting diagram suggests a separation of phonons into three types: (i) pure inorganic modes $(I)$, at $x=0$, confined around $250 \mathrm{~cm}^{-1}$; (ii) a large number of hybrid modes $(H)$, with $x \sim 0.5$, having energy below $200 \mathrm{~cm}^{-1}$, and (iii) organic modes $(O)$, at $x=1$, above $300 \mathrm{~cm}^{-1}$. The $(I)$ modes are exactly those photoexcited Jahn-Teller modes mentioned before, thus contribute to the SC ensemble. An estimate of the $I$ modes contribution to the heat capacity ${ }^{1}$ is shown in the inset of Fig. 5. The plot indicates that the contribution of the $I$ modes to $C_{\text {total }}$ is less than $10 \%$, while that of the $H$ modes is approximately $80 \%$.

The only unaccounted modes in the phonon diagram of Fig. 5 are the acoustic-purely $\mathrm{H}$, with $x=0.5$ - phonons $\left[\omega_{a}(\gamma=0)=0\right]$, as well as all those phonons with strong temperature variations at $T_{C}$, like the $\alpha$ mode in Ref. [15]. Acoustic modes, and more in general HGV modes, are

${ }^{1}$ Partial heat capacities are calculated with the Einstein model, in which each optical mode of energy $\omega_{i}$ contributes $k_{B}\left(\frac{\hbar \omega_{i}}{k_{B} T}\right)^{2} \frac{e^{\hbar \omega_{i} / K_{B} T}}{\left(e^{\hbar \omega_{i} / K_{B} T}-1\right)^{2}}$ to the total heat capacity.

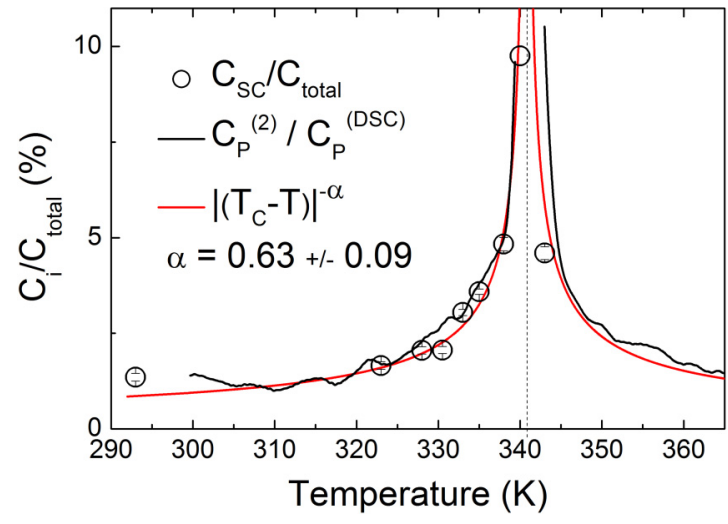

FIG. 6. (Color online) Comparison between $C_{S C}$, as obtained by scaling $g$, and the continuous diverging component of $C_{P}, C_{P}^{(2)}$. The divergence is fitted with a power law to extract the critical exponent of the phase transition at $340 \mathrm{~K}$.

responsible for the thermal transport in any material. Since optical $I$ modes can only decay to such HGV phonons ${ }^{2}$ we expect such HGV modes to contribute to the SC ensemble too. Since there are few HGV modes, ${ }^{3}$ it is reasonable to assume that

$$
C_{S C}=\left(C_{I}+C_{H G V}\right) \ll\left(C_{H}+C_{O}\right)=C_{U}
$$

(see also inset of Fig. 5), and Eq. (7) can be simplified to

$$
\tau \simeq \frac{C_{S C}}{g} .
$$

Equation (9) implies that the measured relaxation time $\tau$ is only function of the smallest subsystem heat capacity $C_{S C}$ and of the thermal coupling constant $g$. In particular, it is now clear that the divergence of $\tau$ is due to the divergence of a component of the heat capacity. Interestingly, as measured from thermal calorimetry, there is only one component of $C_{\text {total }}$, $C_{P}^{(2)}{ }^{4}$ which diverges. This suggests that $C_{P}^{(2)}=C_{S C}$, and that it is possible to determine the thermal coupling constant $g$. In fact, as shown in Fig. 6, the agreement between $C_{S C}=g \tau$ and $C_{P}^{(2)}$ after proper scaling of $g$ is very good. The resulting estimate of the thermal coupling constant is $g=0.410^{17}$ $\mathrm{W} / \mathrm{Km}^{3}$, interestingly very close to the electron-phonon coupling constant measured in metals $[25,26]$. Note that the fact that $\operatorname{Max}\left(C_{S C} / C_{\text {total }}\right)<10 \%$ guarantees the previously made assumption that $C_{S C} \ll C_{U}$ [Eq. (8)], even in proximity of the critical temperature.

\footnotetext{
${ }^{2}$ As observed in Ziman [27], optical phonons can decay mostly via two acoustic phonons $o \rightarrow a+a$ ( $o$ as optical and $a$ as acoustic phonons) since all other processes are strongly suppressed by energy and $k$-vector conservations, and selection rules. Also high $k$-vector optical phonons, which might be created in the absorption process, have high probability of decaying to low-energy modes, via umklapp processes [28].

${ }^{3}$ It should be noted that acoustic phonons at the Brillouin zone boundary are actually not $\mathrm{HGV}$ phonons, as the dispersion is flat at the momentum region.$$
{ }^{4} C_{P}^{(2)}=c_{P}^{(2)} \rho V \text {. }
$$ 


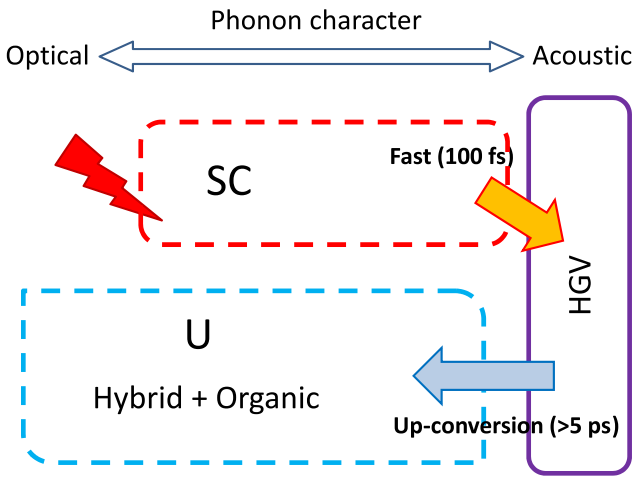

FIG. 7. (Color online) Energy relaxation diagram: light excites $\mathrm{Cu}$ electrons, which relax in very short time scales to strongly coupled (SC) modes, like the Jahn-Teller $\mathrm{Cu}-\mathrm{Cl}$ modes; pure $\mathrm{Cu}-\mathrm{Cl}$ inorganic modes relax than to high group velocity $(\mathrm{HGV})$ phonons $(<1 \mathrm{ps})$. Thermal equilibrium is established by energy transfer from $\mathrm{HGV}$ phonons to higher-energy optical, mostly hybrid, phonons.

The coupling constant $g$ quantifies the up-conversion scattering rate from HGV low-energy phonons to higherenergy optical phonons. Let us first consider the microscopic processes involved during the first picosecond after the photoexcitation. Photoexcited $\mathrm{Cu}$ electrons are strongly coupled to optical JT modes ( $I$ modes in Fig. 5). As observed in Ziman [27], optical phonons can decay mostly via two acoustic phonons $o \rightarrow a+a$ ( $o$ as optical and $a$ as acoustic phonons) since all other processes are strongly suppressed by energy and $k$-vector conservations, and selection rules. Also high $k$-vector optical phonons, which might be created in the absorption process, have high probability of decaying to low-energy modes, via umklapp processes [28]. Once JT modes fully relax to $\mathrm{HGV}$ phonons $(<1 \mathrm{ps})$ the inverse up-conversion processes $a+a \rightarrow o$ take place, until thermal equilibrium is established by energy transfer to optical $\mathrm{H}$ modes. A summary of the dynamics and time scales of the thermalization processes is shown in Fig. 7. Note that the process $o \rightarrow a+a$ is more probable than the inverse one because of the Fermi "Golden rule" [27,29]: $W(i \rightarrow f)=\frac{2 \pi}{\hbar}\left|\left\langle f\left|H^{\prime}\right| i\right\rangle\right|^{2} D_{f}(E)$, where $i$ and $f$ are the initial and final states, $W(i \rightarrow f)=1 / \tau_{i f}$ represent the transition probability (inverse of the decay rate $\left.\tau_{i f}\right), H^{\prime}$ is the time-dependent perturbation hamiltonian and $D_{f}(E)$ the density of final states. While $D_{a+a \rightarrow o}(E)$ represents only a single probability point in the phase space, $D_{o \rightarrow a+a}(E)$ represents the curve, a single variable integral in $k$ space. In summary, the thermal coupling constant $g$, measured by TRB experiments, represents the phonon scattering process $a+a \rightarrow o$, and more in specific the thermalization between HGV phonons to higher-energy optical hybrid modes.
The slowing down is caused by an $H$ mode which changes its phonon character, from $o$ to $a$, while approaching the phase transition and has diverging heat capacity. Strictly speaking such mode below $T_{C}$ is an optical $U$ mode and continuously transforms to a quasi-acoustic HGV mode approaching $T_{C}$. This is perfectly in line with soft mode behavior, where anomalous bending of the dispersion is observed at the critical temperature, causing both increase of group velocity and the increase of the heat capacity. Finally at $T_{C}$ the mode holds part of the heat, slowing down the up-conversion.

The divergence of $C_{S C}$ is in agreement with Raman spectroscopy measurements [15], where low-frequency modes are observed to soften at the critical temperature. The softening of vibrational modes causes, in general, changes of the frequency spectrum $\mathfrak{D}(k)$ [27], which strongly influences the heat capacity. Additionally the decrease of the soft-mode energy $\omega(k)$ (at some $k$ vector of the Brillouin space) is, in general, partnered by an increase of the phonon group velocity $\frac{\partial \omega(k)}{\partial k}$. Thus, in proximity of the ferroelectric phase transition, the "soft" vibrational modes gain the character of HGV modes, thus contributing to the SC ensemble. Additionally, because of the collapse of $\omega(k)$, the "soft" modes might also cause the divergence of heat capacity term $c_{P}^{(2)}$. Assuming direct coupling between the divergence of $C_{S C}$ and the soft-mode behavior described in Raman spectroscopy it is possible to derive, based on scaling laws hypothesis [30], all critical exponents of the phase transition (see Ref. [21]). By fitting $C_{S C}$ with a power law (see Fig. 6) it is found that $\alpha=0.63 \pm 0.09$.

\section{CONCLUSION}

We measure the thermal coupling constant of the upconversion processes from low-energy high group velocity phonons to optical high-energy phonons. The thermal coupling constant of the process $a+a \rightarrow o$ is $g \sim 0.410^{17} \mathrm{~W} / \mathrm{Km}^{3}$, very close to the electron-phonon coupling in metals.

The method presented here combines different techniques, namely time-resolved optical experiments, DFT calculation, and DSC measurements. It is shown how time constants are strongly influenced by changes of heat capacity of the system, in particular in proximity of phase transitions. At the same time a comparative procedure is described which allows for the determination of fundamental parameters like the thermal coupling constant $g$ and partial heat capacity contributions.

\section{ACKNOWLEDGMENTS}

We acknowledge B. Noheda Pinuaga for fruitful discussions. We would like to thank G. Alberda van Ekenstein for the measurements, and relative discussion, on the heat capacity.
[1] S. Wolfram, A New Kind of Science, General Science (Wolfram Media, Champaign, IL, 2002), p. 1019.

[2] R. Peierls, Quantum Theory of Solids (Oxford University Press, New York, 2001).

[3] S. Das Sarma, S. Adam, E. Hwang, and E. Rossi, Rev. Mod. Phys. 83, 407 (2011).
[4] J. Baringhaus, M. Ruan, F. Edler, A. Tejeda, M. Sicot, TalebIbrahimi Amina, A.-P. Li, Z. Jiang, E. H. Conrad, C. Berger, C. Tegenkamp, and W. A. de Heer, Nature (London) 506, 349 (2014).

[5] T. Popmintchev, M.-C. Chen, D. Popmintchev, P. Arpin, S. Brown, S. Ališauskas, G. Andriukaitis, T. Balčiunas, O. D. 
Mücke, A. Pugzlys, A. Baltuška, B. Shim, S. E. Schrauth, A. Gaeta, C. Hernández-García, L. Plaja, A. Becker, A. JaronBecker, M. M. Murnane, and H. C. Kapteyn, Science 336, 1287 (2012).

[6] M. E. Siemens, Q. Li, R. Yang, K. A. Nelson, E. H. Anderson, M. M. Murnane, and H. C. Kapteyn, Nat. Mater. 9, 26 (2009).

[7] A. Eschenlohr, M. Battiato, P. Maldonado, N. Pontius, T. Kachel, K. Holldack, R. Mitzner, A. Fölisch, P. M. Oppeneer, and C. Stamm, Nat. Mater. 12, 332 (2013).

[8] M. Lax, P. Hu, and V. Narayanamurti, Phys. Rev. B 23, 3095 (1981).

[9] P. Klemens, Phys. Rev. 148, 845 (1966).

[10] D. Xiong, Y. Zhang, and H. Zhao, Phys. Rev. E 88, 052128 (2013).

[11] N. J. Ekins-Daukes, I. Ballard, C. D. J. Calder, K. W. J. Barnham, G. Hill, and J. S. Roberts, Appl. Phys. Lett. 82, 1974 (2003).

[12] J. Shiomi and S. Maruyama, Phys. Rev. B 73, 205420 (2006).

[13] A. O. Polyakov, A. H. Arkenbout, J. Baas, G. R. Blake, A. Meetsma, A. Caretta, P. H. M. van Loosdrecht, and T. T. M. Palstra, Chem. Mater. 24, 133 (2012).

[14] A. Caretta, R. Miranti, A. H. Arkenbout, A. O. Polyakov, A. Meetsma, R. Hidayat, M. O. Tjia, T. T. M. Palstra, and P. H. M. V. Loosdrecht, J. Phys. Condens. Matter 25, 505901 (2013).

[15] A. Caretta, R. Miranti, R. W. A. Havenith, E. Rampi, M. C. Donker, G. R. Blake, M. Montagnese, A. O. Polyakov, R. Broer, T. T. M. Palstra, and P. H. M. van Loosdrecht, Phys. Rev. B 89, 024301 (2014).
[16] D. Mitzi, J. Chem. Soc., Dalton Trans. 1 (2001).

[17] W. E. Estes, D. B. Losee, and W. E. Hatfield, J. Chem. Phys. 72, 630 (1980).

[18] L. J. de Jongh and A. R. Miedema, Adv. Phys. 23, 1 (1974).

[19] A. Caretta, M. C. Donker, A. O. Polyakov, T. T. M. Palstra, and P. H. M. van Loosdrecht, Phys. Rev. B 91, 020405 (2015).

[20] P. Gill, S. Sauerbrunn, and M. Reading, J. Therm. Anal. 40, 931 (1993).

[21] A. Caretta, Ph.D. Thesis, University of Groningen, The Netherlands, 2014.

[22] A. H. Arkenbout, Ph.D. Thesis, University of Groningen, 2010.

[23] G. L. Eesley, Phys. Rev. B 33, 2144 (1986).

[24] H. E. Elsayed-Ali, T. B. Norris, M. A. Pessot, and G. A. Mourou, Phys. Rev. Lett. 58, 1212 (1987).

[25] S. D. Brorson, A. Kazeroonian, J. S. Moodera, D. W. Face, T. K. Cheng, E. P. Ippen, M. S. Dresselhaus, and G. Dresselhaus, Phys. Rev. Lett. 64, 2172 (1990).

[26] P. B. Allen, Phys. Rev. Lett. 59, 1460 (1987).

[27] J. M. Ziman, Electrons and Phonons (Oxford University Press, London, 1962).

[28] S. Zakharov, Solid State Commun. 66, 1251 (1988).

[29] P. Aynajian, Electron-Phonon Interaction in Conventional and Unconventional Superconductors, Springer Theses (Springer, New York, 2011).

[30] H. E. Stanley, Rev. Mod. Phys. 71, S358 (1999). 